\title{
Surgical and pathological outcomes of laparoscopic surgery for transverse colon cancer
}

\author{
Y. S. Lee • I. K. Lee • W. K. Kang • H. M. Cho • \\ J. K. Park • S. T. Oh • J. G. Kim • Y. H. Kim
}

Accepted: 4 March 2008 / Published online: 1 April 2008

(C) The Author(s) 2008

\begin{abstract}
Purpose Several multi-institutional prospective randomized trials have demonstrated short-term benefits using laparoscopy. Now the laparoscopic approach is accepted as an alternative to open surgery for colon cancer. However, in prior trials, the transverse colon was excluded. Therefore, it has not been determined whether laparoscopy can be used in the setting of transverse colon cancer. This study evaluated the peri-operative clinical outcomes and oncological quality by pathologic outcomes of laparoscopic surgery for transverse colon cancer.

Materials and methods Analysis of the medical records of patients who underwent laparoscopic colorectal resection from August 2004 to November 2007 was made. Computed tomography, barium enema, and colonoscopy were performed to localize the tumor preoperatively. Extended right hemicolectomy, transverse colectomy, and extended left hemicolectomy were performed for transverse colon cancer. Surgical outcomes and pathologic outcomes were compared between transverse colon cancer (TCC) and other site colon cancer (OSCC).

Results Of the 312 colorectal cancer patients, 94 patients underwent laparoscopic surgery for OSCC, and 34 patients underwent laparoscopic surgery for TCC. Patients with
\end{abstract}

Y. S. Lee $\cdot$ I. K. Lee - W. K. Kang $\cdot$ H. M. Cho · J. K. Park S. T. Oh· J. G. Kim • Y. H. Kim

Department of Surgery, The Catholic University of Korea,

Seoul, South Korea

Y. S. Lee $(\bowtie)$

Department of Surgery, Our Lady of Mercy Hospital,

\#665, Bupyung-6-dong, Bupyunggu,

Incheon 403-720, South Korea

e-mail: yslee@catholic.ac.kr
TCC were similar to patients with OSCC in age, gender, body mass index, operating time, blood loss, time to pass flatus, start of diet, hospital stay, tumor size, distal resection margin, proximal resection margin, number of lymph nodes, and radial margin. One case in TCC and three cases in OSCC were converted to open surgery.

Conclusions Laparoscopic surgery for transverse colon cancer and OSCC had similar peri-operative clinical and acceptable pathological outcomes.

Keywords Laparoscopy · Transverse colon · Colon cancer

\section{Introduction}

Since its first report [1], laparoscopic colon surgery has been controversial with regard to its use for colorectal cancer. Several prospective randomized trials including the COLOR and CLASSIC studies have demonstrated that laparoscopicassisted surgery for colorectal cancer resulted in a shorter hospital stay, reduced analgesic use, and earlier recovery to bowel movement [2-6]. Moreover, the COST study established the long-term oncological safety of laparoscopicassisted surgery for colon cancer, and currently the laparoscopic approach is accepted as an alternative to open surgery for colon cancer [7]. However, transverse colon cancer was excluded from prior randomized controlled trials. The reasons for exclusion of transverse colon cancer include the following: difficulty in deciding the appropriate operative procedure and extent of lymph node dissection, as well as technical difficulties with the laparoscopic identification, ligation, and lymph node dissection around the middle colic vessels. Therefore, there continues to be debate on whether to use laparoscopic surgery for transverse colon cancer. 


\section{Materials and methods}

Between August 2004 and November 2007, the medical records of all patients who underwent laparoscopic surgery for colorectal cancer were reviewed. Laparoscopic colorectal cancer resection was started August 2004 in our clinic. Pathologic confirmation, colonoscopy, barium enema, computed tomography (CT), ultrasonography, and chest PA were performed for diagnosis in all patients preoperatively. All patients with colorectal adenocarcinoma admitted to our clinic were considered for laparoscopic surgery. Exclusion criteria for laparoscopic surgery were as follows: (1) patients with colorectal cancer obstruction and failure of stent insertion, (2) patients with colorectal cancer perforation requiring emergency surgery, (3) patients with $\mathrm{T} 4$ colorectal cancer lesion that could not be resected laparoscopically, and (4) patients with compromised cardio-pulmonary function in whom pneumoperitoneum under general anesthesia was contraindicated. In this study, transverse colon cancer was defined as lesions between the hepatic flexure and splenic flexure in the colon, requiring ligation of the middle colic vessels at their origin. CT or barium enema was performed in all patients with colon cancer preoperatively for localization of the tumor. If radiological localization was unclear, preoperative colonoscopic Indian ink tattooing or endoscopic clipping was performed.

The procedure used for the transverse colon cancer was chosen based on the location of the tumor. A tumor located at the hepatic flexure or within $10 \mathrm{~cm}$ distal to hepatic flexure was treated by an extended right hemicolectomy, and a tumor located at the splenic flexure or within $10 \mathrm{~cm}$ proximal to the splenic flexure was treated by an extended left hemicolectomy. A tumor located between the above two lesions was treated by a transverse colectomy. An extended right hemicolectomy was defined as lymphadenectomy simultaneously with ligation of ileocolic, right colic, and middle colic vessels at their origins; an extended left hemicolectomy was defined as lymphadenectomy simultaneously with ligation of left colic and middle colic vessels at their origins. A transverse colectomy was defined as lymphadenectomy simultaneously with ligation of middle colic vessels at their origins. Extra-corporeal anastomosis was performed in all cases of laparoscopic surgery for transverse colon cancer. All patients started a diet after passing flatus. To evaluate the postoperative surgical outcomes and oncological quality of laparoscopic surgery for transverse colon cancer, we compare the age, gender, body mass index (BMI), operating time, blood loss, time to passing flatus, time to start of diet, hospital stay, surgical morbidity, surgical mortality, conversion to open surgery, tumor size, distal resection margin, proximal resection margin, radial margin, and number of harvested lymph nodes between the transverse colon cancer group (TCC) and other site colon cancer group (OSCC). In this study, a single surgeon (YS Lee) performed all operations. Comparisons between the two groups were made by applying the independent samples $t$ test and $\chi^{2}$ test. Differences were considered to be significant for $P$ value $<0.05$.

\section{Results}

Postoperative clinical outcomes

Three hundred and twelve patients underwent colorectal cancer resection in our clinic during these periods. Among them, 44 patients underwent conventional open surgery according to exclusion criteria (five cases of stent failure or colon obstruction, five cases of emergency operation, 12 cases of far advanced tumor, 12 cases of recurrent cancer operation, seven cases of patient's refusal, and three cases for old age with high risk for pneumoperitoneum). There was no transverse colon cancer in the conventional open surgery group. A total of 268 patients underwent laparoscopic resection for colorectal cancer. Of the 268 patients, 140 patients who underwent laparoscopic resection for rectal cancer were excluded in this study. Finally, 128 patients who underwent laparoscopic resection for colon cancer were enrolled in this study. Of the 128 patients, 34 patients had transverse colon cancer, counting for $10.8 \%$ of total colorectal cancers, and 94 patients had other sites of colon cancer. In the TCC group, extended right hemicolectomy was performed in 18 cases, transverse colectomy was performed in eight cases, and extended left hemicolectomy was performed in eight cases. In the OSCC group, right hemicolectomy was performed in 38 cases, left hemicolectomy was performed in five cases, and anterior resection was performed in 51 cases. There were no statistical differences in age, gender distribution, BMI, operating time, intraoperative blood loss, time to flatus, time to start of diet, and hospital stay between patients with TCC and OSCC (Table 1). Four patients in the OSCC group had a major complication, one had colon injury, and two had anastomosis leak. A simple laparoscopic closure was performed in the case of colon injury, and two patients with anastomosis leak underwent re-operation. Minor complications that occurred in four cases in the OSCC group (one case of ileus, two cases of port site minor infection, and one case of urinary retention) and two cases in the TCC group (one case of ileus and one case of atelectasis) were treated successfully conservatively. One case among the TCC group and three cases in the OSCC group were converted to open surgery; all of these converted cases were due to tumor-related factors, T4 lesion, or huge tumor. There was no surgical mortality in this study. 
Table 1 Clinical characteristics of patients OSCC other site colon cancer, NS not significant

${ }^{\mathrm{a}}$ Values are ranges.

\begin{tabular}{lccc}
\hline & TCC $(N=34)$ & OSCC $(N=94)$ & $P$ value \\
\hline Age (years) & $64.1 \pm 11.3$ & $62.5 \pm 12.1$ & $\mathrm{NS}$ \\
Sex (M:F) & $15: 19$ & $46: 48$ & $\mathrm{NS}$ \\
Body mass index $\left(\mathrm{kg} / \mathrm{m}^{2}\right)$ & $23.5 \pm 3.0$ & $24.0 \pm 3.1$ & $\mathrm{NS}$ \\
Operating time (min) & $211.1 \pm 52.2(140-360)^{\mathrm{a}}$ & $220.4 \pm 94.3(60-620)^{\mathrm{a}}$ & $\mathrm{NS}$ \\
Blood loss (ml) & $100.0 \pm 83.8(0-400)^{\mathrm{a}}$ & $114.2 \pm 145.2(0-700)^{\mathrm{a}}$ & $\mathrm{NS}$ \\
Time to pass flatus (days) & $2.8 \pm 0.8$ & $2.6 \pm 1.0$ & $\mathrm{NS}$ \\
Diet start (days) & $4.2 \pm 1.8$ & $4.2 \pm 2.8$ & $\mathrm{NS}$ \\
Hospital stay (days) & $11.4 \pm 4.1$ & $11.2 \pm 6.0$ & $\mathrm{NS}$ \\
\hline
\end{tabular}

Oncological quality by pathologic outcomes

There were no statistical differences in the tumor size, proximal resection margin, distal resection margin, radial margin, and number of harvested lymph nodes between patients with TCC and OSCC (Table 2).

\section{Discussion}

Since laparoscopic colon resection was first reported in 1991 [1], laparoscopic surgery has been widely employed for various benign colorectal disease such as benign mass, diverticular disease, inflammatory bowel disease, rectal prolapse, and now increasingly for colorectal malignant disease. Evidences of the safety and efficacy of the laparoscopic surgery for colorectal cancer had been reported from several prospective randomized controlled studies and meta-analysis of several trials, which favored the laparoscopic surgery for colorectal cancer over conventional open surgery due to its many short-term benefits such as shorter hospital stay, reduced use of analgesics, and earlier recovery of bowel movements [2-6, 8-13]. Eventually, long-term oncological safety of laparoscopic colon cancer resection was established, and the laparoscopic approach was accepted as an alternative to open surgery for colon cancer by the COST study [7].

Transverse colon cancer occurs in about $10 \%$ of cases of colorectal cancer, and it often presents a challenge for the choice of the best surgical procedure based on the location of the tumor and extent of lymph node dissection. There could also be technical difficulties with laparoscopic identification, ligation, and lymph node dissection around the middle colic vessels depending on the surgeon's experience. Because of these reasons, transverse colon cancer was excluded from almost every prior prospective randomized trial. Therefore, there is continued debate over the validity of laparoscopic surgery for transverse colon cancer. The major controversy about laparoscopic surgery for transverse colon cancer lies on whether or not it is feasible to perform sufficient extent of lymph node dissection around the middle colic artery laparoscopically. As experiences of laparoscopic surgery are accumulating and surgical techniques and instruments are developing, we consider that the extent of laparoscopic lymph node dissection for transverse colon cancer is not less than the extent of conventional lymph node dissection for transverse colon cancer.

Some of the difficulties with laparoscopic surgery for transverse colon cancer that need to be resolved include the following: First, precise localization of carcinoma of the transverse colon is important; this is because the extent of resection and lymph node dissection depends on the location of the tumor in the transverse colon. Diminished tactile guidance can make localization of the tumor in the transverse colon more difficult in small tumor. There are a number of methods used to localize the tumor. Preoperative barium enema is useful for localization of large and advanced tumor, but radiological localization is inconclusive and difficult in early cancer. In such cases, colonoscopic tattooing with Indian ink or placing endoscopic clips is very effective. Using endoscopic clip at the time of preoperative colonoscopy and using fluoroscopy

Table 2 Pathological outcomes of patients

\begin{tabular}{lccc}
\hline & TCC $(N=34)$ & OSCC $(N=94)$ & $P$ value \\
\hline Tumor size $(\mathrm{cm})$ & $5.2 \pm 2.5(0.7-11)^{\mathrm{a}}$ & $4.6 \pm 2.4(0.1-14)^{\mathrm{a}}$ & NS \\
PRM $(\mathrm{cm})$ & $19.5 \pm 10.2(10.0-25.0)^{\mathrm{a}}$ & $15.3 \pm 11.0(4.2-25.0)^{\mathrm{a}}$ & NS \\
DRM $(\mathrm{cm})$ & $13.9 \pm 6.9(6.0-25.0)^{\mathrm{a}}$ & $11.6 \pm 5.3(3.0-32.0)^{\mathrm{a}}$ & $\mathrm{NS}$ \\
No. of lymph nodes & $24.4 \pm 11.7(3-59)^{\mathrm{a}}$ & $21.1 \pm 8.4(3-59)^{\mathrm{a}}$ & $\mathrm{NS}$ \\
Radial margin $(\mathrm{cm})$ & $0.9 \pm 0.8(0-3.0)^{\mathrm{a}}$ & $1.0 \pm 0.9(0-3.5)^{\mathrm{a}}$ & NS \\
\hline
\end{tabular}

TCC Transverse colon cancer, OSCC other site colon cancer, DRM distal resection margin, PRM proximal resection margin, NS not significant ${ }^{\text {a }}$ Values are ranges. 
for localization, the clip migration could be the main problem, and using fluoroscopy in the operating theater is troublesome and time consuming. However, in the case of hepatic flexure or splenic flexure colon cancer, by checking X-ray just after placing the endoscopic clip, we could precisely localize both flexure tumors easily. Intraoperative colonoscopy can be used for localization of the tumor, but it is also time consuming, and moreover, it can cause colonic insufflation that makes laparoscopic surgery difficult [14]. Properly placed tattoos are long lasting and can be placed at the time of diagnostic colonoscopy [15]. In the event of tattoo failure, one can use intraoperaitve colonoscopy for localization easily. In this study, we performed preoperative colonoscopic tattoo in three cases to localize the mid transverse colon cancer, colonoscopic clipping in three cases of hepatic flexure colon cancer, and barium enema in the other cases. There was no case with non-localization. We think that colonoscopic tattooing is effective in small mid transverse colon cancer, and endoscopic clipping is effective in small hepatic or splenic flexure colon cancer. Second is the laparoscopic identification, ligation, and lymph node dissection around the middle colic vessels. Fujita et al. reported laparoscopic techniques that can be used for identification of middle colic vessel. They suggested that the ventral aspect of the caudal portion of superior mesenteric vein be exposed, and the exposed vessel brought cephalad toward the caudal portion of the pancreas, with identification of the middle colic vessels [16]. Baca et al. introduced the 'window technique' for lymphadenectomy with simultaneous resection of the vascular stem [17]. Ichihara et al. introduced the technique of rotation of mesocolon to identify middle colic artery [18]. The authors have identified the middle colic vessels using surgical techniques introduced by Fujita in most of the cases, and the in case of slender patients we could directly identify pulsating middle colic artery by stretching the transverse mesocolon by traction of both ends of the transverse colon by the first assistant. Identifying middle colic vessels, excessive traction of the transverse mesocolon by the first assistant may cause tearing of the vein and bleeding. Moreover, the length of the gastrocolic trunk of Henle is relatively short, and attempt to control bleeding from the gastrocolic trunk of Henle can injure the superior mesenteric vein [19]. It is especially important for a laparoscopic colorectal surgeon to recognize the variable drainage of the right colic and middle colic vein to the gastrocolic trunk of Henle and to have a precise knowledge of superior right colic vein anatomy. Third is the low incidence of transverse colon cancer. Consecutive cases are needed for overcoming the learning curve. Therefore, it might take surgeons longer time to become experienced in the techniques used for laparoscopic transverse colon cancer resection.
In this study, there were no significant differences between patients with TCC and OSCC in terms of operating time, blood loss, resection margin, and number of lymph nodes. Schlachta et al. reported that the operating time was longer in the TCC group compared to the OSCC group, and the number of harvested lymph nodes in the TCC group was greater than in the OSCC group because of the extended resection and larger specimens, as well as the need to attend to the middle colic vessels in patients with TCC [20]. However, in this study, there was no statistical difference in operating time and number of harvested lymph nodes, as well as the surgical outcomes between the two groups. We think that operating time could be similar in the TCC and OSCC groups in experienced hands, and actually, the extent of lymph node dissection in transverse colon cancer is not too wide than that of other site colon cancer, so there were no statistical differences in pathological outcomes between the two groups in this study.

There was one case of colon injury proximal to anastomosis caused by electrocautery during the operation in the OSCC group; this was treated by a laparoscopic simple closure on postoperative day 3. Three cases of anastomosis leak, one case of right hemicolectomy, and two cases of anterior resection occurred in the OSCC group. A patient with anastomosis leak was diagnosed by clinical symptoms or sign, fecal or purulent discharge from drain, fever, leukocytosis, and peritoneal irritation sign. Radiologic study using a water-soluble dye was not performed in this study. Of the three cases, one patient with a leak of anterior resection was treated conservatively successfully, and two patients with leak cases underwent laparoscopic reoperation. All other minor complications were successfully treated conservatively.

Conversion to open surgery occurred in one case in the TCC group and in three cases in the OSCC group; these differences were not significant. Factors related to the tumor were the cause of conversion to open surgery. One case in the TCC group was converted to open surgery due to T4 lesion in the transverse colon cancer that invaded the anterior wall of the stomach. In the OSCC group, one case with a larger tumor that was hard to handle by laparoscopy, and two cases of sigmoid colon cancer that invaded the uterus were converted to open surgery. However, T4 colon cancer is not a contraindication for laparoscopy if en bloc resection could be performed with the laparoscopy. In those cases converted to open surgery, laparoscopic en bloc resection was impossible.

This study has some weak points. First, the number of patients with transverse colon cancer is too small, making up $10 \%$ of the total colorectal cancer resection and $12 \%$ of the total laparoscopic colorectal resection in this study. The second is that although the data in this study were collected 
prospectively, the data were not derived from a larger case series and this study was not randomized controlled. The third is that the mean follow-up period was too short to evaluate the oncological outcomes (15.9 months; range 140 months). We think that large-scale prospective controlled trials and long-term analysis are mandatory to overcome these limitations and confirm the oncological safety of laparoscopic transverse colon cancer surgery. We are going to report a long-term analysis after a long-term follow-up involving more cases prospectively.

\section{Conclusions}

The results of this study show no significant differences with regard to surgical outcomes and oncological quality by pathologic outcomes between patients in the OSCC and TCC groups. Further investigations with large-scale prospective studies and long-term analysis of laparoscopic surgery for transverse colon cancer are mandatory to establish the oncological safety of laparoscopic surgery for transverse colon cancer.

Open Access This article is distributed under the terms of the Creative Commons Attribution Noncommercial License which permits any noncommercial use, distribution, and reproduction in any medium, provided the original author(s) and source are credited.

\section{References}

1. Jacobs M, Verdeja JC, Goldstein HS (1991) Minimally invasive colon resection (laparoscopic colecotomy). Surg Laparosc Endosc $1: 144-150$

2. Stage JG, Schulze S, Moller P, Overgaard H, Andersen M, Rebsdorf-Pedersen VB, Nielsen HJ (1997) Prospective randomized study of laparoscopic versus open colonic resection for adenocarcinoma. Br J Surg 84:391-396

3. Milsom JW, Bohm B, Hammerhofer KA, Fazio V, Steiqer E, Elson P (1998) A prospective randomized trial comparing laparoscopic versus conventional techniques in colorectal cancer surgery: a preliminary report. J Am Coll Surg 187:46-54

4. Leung KL, Kwok SP, Lam SC, Lee JF, Yiu RY, Ng SS, Lai PB, Lau WY (2004) Laparoscopic resection of rectosigmoid carcinoma: prospective randomized trial. Lancet 363:1187-1192
5. Guillou PJ, Quirke P, Thorpe H, Walker J, Jayne DG, Smith AM, Heath RM, Brown JM, MRC CLASICC Trial Group (2005) Short-term endpoints of conventional versus laparoscopic assisted surgery in patients with colorectal cancer (MRC CLASICC trial): multicentre, randomized controlled study. Lancet 365: $1718-1726$

6. The Colon Cancer Laparoscopic or Open Resection Study Group (2005) Laparoscopic surgery versus open surgery for colon cancer: short-term outcomes of a randomized trial. Lancet Oncol $6: 477-484$

7. The Clinical Outcomes of Surgical Therapy Study Group (2004) A comparison of laparoscopically assisted and open colectomy for colon cancer. N Engl J Med 350:2050-2059

8. Abraham NS, Byrne CM, Young JM, Solomon MJ (2007) Metaanalysis of non-randomized comparative studies of the short-term outcomes of laparoscopic resection for colorectal cancer. ANZ J Surg 77:508-516

9. Kamyar K, Margherita C, Forough F, Mehran A (2007) Laparoscopic surgery for colon cancer: a systemic review. Can J Surg 50:48-57

10. Tjandra JJ, Chan MKY (2006) Systemic review on the short-term outcomes of laparoscopic resection for colon and rectosigmoid cancer. Colorectal Dis 8:375-388

11. Breukink S, Pierie J, Wiggers T (2006) Laparoscpic versus open total mesorectal excision for rectal cancer. Cochrane Database Syst Rev 18:CD005200

12. Abraham NS, Young JM, Solomon MJ (2004) Meta-analysis of short-term outcomes after laparoscopic resection for colorectal cancer. Br J Surg 91:1111-1124

13. Jackson TD, Kaplan GG, Arena G, Page JH, Rogers SO Jr (2007) Laparoscopic versus open resection for colorectal cancer: a metaanalysis of oncologic outcomes. J Am Coll Surg 204:439-446

14. Feingold DL, Addona T, Forde KA, Arnell TD, Carter JJ, Huang EH, Whelan RL (2004) Safety and reliability of tattooing colorectal neoplasm prior to laparoscopic resection. J Gastrointest Surg 8:543-546

15. Fennerty MB, Sampliner RE, Hixson LJ, Garewal HS (1992) Effectiveness of India ink as a long-term colonic mucosal marker. Am J Gastroenterol 87:79-81

16. Fujita J, Uyama I, Suqioka A, Komori Y, Matsui H, Hasumi A (2001) Laparoscopic right hemicolectomy with radical lymph node dissection using the no-touch isolation technique for advanced colon cancer. Surg Today 31:93-96

17. Baca I, Perko Z, Bokan I, Mimica Z, Petricevic A, Druijanic N, Situm M (2005) Technique and survival after laparoscopically assisted right hemicolectomy. Surg Endosc 19:650-655

18. Ichihara T, Takada M, Fukumoto S, Kuroda Y (2004) Lymphadenectomy along the middle colic artery in laparoscopic resection of transverse colon. Hepatogastroenterology 51:455-456

19. Ignjatovic D, Stimec B, Finjor T, Bergamaschi R (2004) Venous anatomy of the right colon: three-dimensional topographic mapping of the gastrocolic trunk of Henle. Tech Coloproctol 8:19-22

20. Schlachta CM, Mamazza J, Poulin EC (2006) Are transverse colon cancers suitable for laparoscopic resection. Surg Endosc 21:396-399 2007 